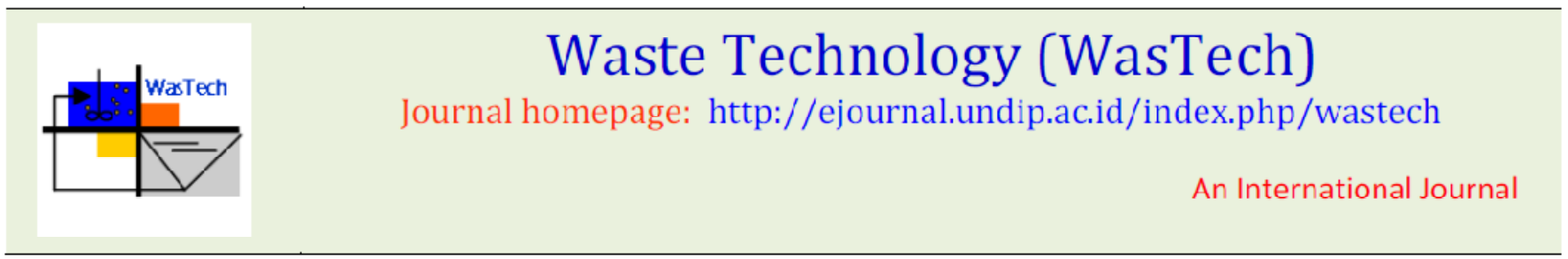

\title{
Bioacoustic spectral whistle sound and behaviour of male dolphin bottle nose (Tursiops aduncus) at Safari Park Indonesia, Cisarua Bogor
}

\author{
Muhammad Zainuddin Lubis ${ }^{1 *}$, Sri Pujiyati ${ }^{2}$, Totok Hestirianoto ${ }^{2}$ \\ ${ }^{1}$ Postgraduate Student Department of Marine Science and Technology, Faculty of Fisheries and Marine Sciences Bogor \\ Agricultural University Jln. Agatis, Kampus IPB Dramaga Bogor 16680 Indonesia. \\ ${ }^{2}$ LecturerDepartment of Marine Science and Technology, Faculty of Fisheries and Marine Sciences Bogor Agricultural \\ University Jln. Agatis, Kampus IPB Dramaga Bogor 16680 Indonesia. \\ e-mail: lubiszainuddin@gmail.com
}

\begin{abstract}
Whistle sound is generally used for echolocation purposes, while the sound of the blast-beat and whistles play a major role in internal and inter-group communication. The purpose of this research is to know the power spectral patterns and fluctuations in sound based on frequency and Power Spectral Density (PSD) of the sounds produced by dolphins and observing the position of dolphins , Noisy Time Domain (NTD) and behavior using underwater camera, and also comparing between time and sound spectrum. Data recording was taken at the Safari Park of Cisarua Bogor in Indonesia, by taking data in show pool and show pool. The results showed that salinity before feeding time in the show pool with replication 1,2, and 3 has salinity value that is equal to $30 \%$. Data at the show pool before feeding with replications 1,2, and 3 has a salinity of $29 \%$.Power Spectral Density (PSD) of the whistle sound before feeding was different from one another, but it has the same frequency range. The highest intensity values is at sound whistle 3 before feeding time at the show pool with an intensity value of $28.03 \mathrm{~dB} / \mathrm{Hz}$ with frequency interval of $14642 \mathrm{~Hz}-16000 \mathrm{~Hz}$. F-test at the show pool before meals has heterogeneous value. Treat before meals at the show pool has a value of $P<0.001$ and $P<0.001$. Value Noisy Time Domain have differences with each other and have a lot of different sound patterns. In general, there are real differences are evidenced by $F$ test on a range of time at each whistle on physiotherapy and swimming pool show in the time before meals. The position of dolphins in a pool show more dominant and often in the bottom of the pool, the position of the dolphins affect the frequency.

Keywords - Bioacoustic, Whistle sound,Power Spectral Density (PSD), male dolphins bottle nose (Tursiop aduncus).
\end{abstract}

Submission: February 1, 2016

Correction: March 13, 2016

Accepted: April 2, 2016

Doi: http://dx.doi.org/10.12777/wastech.4.1.24-30

[How to cite this article: Lubis, M.Z., Pujiyati, S., and Hestirianoto, T. (2016). Bioacoustic spectral whistle sound and behaviour of male dolphin bottle nose (Tursiops aduncus) at Safari Park Indonesia, Cisarua Bogor. Waste Technology, 4(1),24-30, doi: http://dx.doi.org/10.12777/wastech.4.1.24-30

\section{Introduction}

Currently little is known about the bottlenose dolphin sound has type referred to as yelping (burst ), which is characteristized byc of spectral, temporal, and amplitude as a shrill sound pulse of type the sound of a click type very few researchers have explored. As for early description in literature, most of it is only worth qualitative, that reflects interpretation hearing subjective interpretation and classification hearing in humans [1]. The sound of a click generally used for echolocation, while the sound of a whistle yelping (burst) and playsed a major role in internal communication and across group [2]. Whistles that continues indefinitely , giving signals frequency [3], with various wide emissions of $800 \mathrm{~Hz}$ and $28.5 \mathrm{KHz}$ [4] often there are harmonic components harmonic [3] Dolphin hearing to dolphins rangesd from about $50 \mathrm{~Hz}-150 \mathrm{KHz}$, with somethe variation of additional among species [4]. Previous research about Bioacoustic Characteristic of Male Dolphins Bottle Nose (Tursiops aduncus ) have frequency range of their clicks is at $14.221-15.100 \mathrm{~Hz}$ [5,6] In true dolphins (Delphinidae), tonal sounds are typically referred to as whistles, and are emitted especially during social interactions that involve group cohesion, individual recognition, and recruitment during feeding activities [7] classified whistles of common bottlenose dolphins (Tursiops truncatus) into "signature whistles", (or contact calls) and "variant whistles.' Signature whistles are stereotypic and individualspecific signals that are stable over time and are used for group cohesion.

Bioacoustus is the knowledge that combines biological and acoustics which usually refer to research on the production of sound, dispersion through an elastic 
medium, and acceptance in animals, including humans [8]. But because the science of acoustics is growing rapidly for dolphins, researchers formerly had been exercising records and analysis vocalization [9]. Associating vocalizations with the underwater behavior of dolphins has proved difficult due to the lack of underwater access to animals and life history, sex, and relationship information. Limited reports on the behavior and vocalizations of Atlantic spotted dolphins, have been made [10]. This paper describes analyse and discriminate characteristics whistle sound of male dolphins bottle nose (Tursiops aduncus) in the show pool at safari park Indonesia, Cisarua Bogor. Tools and material used in the research methodology include: hidrofon SQ3 (instrument passive acoustic ), a thermometer hg to measure the temperature of water; refractometer for measuring water salinity, . dolphin EAR 100 hydrophones with serial number DE989505, camera underwater gopro hero $3+$ serves to record the movement of dolphins visually while in the pool.

\section{Research Methods}

\subsection{Power Spectral Density}

The frequency of a wave is naturally determined by the frequency source. The rate of the wave through a medium is determined by the properties of the medium. Once the frequency (f) and speed of sound ( $v$ ) of the wave has been given, then the wavelength $(\lambda)$ has been set. With the relationship $\mathrm{f}=1 / \mathrm{T}$ can be obtained equation (1):

$\lambda=\frac{v}{f}$

Because the study used the speed of sound in the water medium, ie seawater. Then the speed of sound in air is denoted by ( $\mathrm{v}$ ) can be changed with the speed of sound in water that is denoted by (C), so that equation (2) :

$\lambda=\frac{C}{f}$

Power spectral density (PSD) definition as the magnitude of the power per intervals frequency interval, in the form of mathematics [19].

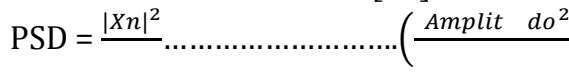

Calculation psd in matlab uses the Welch method welch [11] , namely looking for DFT (Based on accounts with FFT algorithms ), then squaring the value of the magnitude .

\subsection{Recording Whistle}

Sound consists of particle motions that create a pressure wave propagating away from the soundsource at a sound speed determined by the properties of the medium. A sound field is therefore madeup by a particle velocity component ( $v$ ) and a pressure component ( $\mathrm{p})$, and their product definesthe acoustic intensity (I):

$I=p \cdot v$
The particle velocity is given by the pressure divided by the acoustic impedance of the medium. In the acoustic-free field, far from the sound source and any reflecting boundaries, the acoustic impedance is the product of the sound velocity $\mathrm{c}$ and the density of the medium $(p)$. Under those circumstances the sound intensity may therefore be calculated as:

$I=/(\rho c)$

Usually, we express the sound intensity in decibel units:

$10 \log _{10}\left(I / I_{0}\right)$

where $I_{0}$ is the intensity of a plane wave with an rms sound pressure of $1 \mathrm{mPa}$ in water.Accordingly, we quantify the sound pressure as

$$
\mathrm{dB} \text { re } \mu \mathrm{Pa}=20 \log _{10}\left(p / p_{0}\right)
$$

Whistles were extracted from underwater video and sound recordings made between Mei 2015 with video cameras (Gopro Hero 3+) and a SQR 3 hydrophone, flat to $22 \mathrm{kHz}$ with a $-192 \mathrm{~dB}$ re $1 \mu \mathrm{Pa}$. Whistles were assigned to specific individuals when a dolphin was alone in the camera/hydrophone vicinity, in sole proximity $(<1 \mathrm{~m})$ of the camera/hydrophone, or showed simultaneous bubble emissions correlated with a whistle. Although amplitude can be variable in dolphin vocalizations, individuals more than $5 \mathrm{~m}$ away from the recording equipment never emitted whistles as loud as dolphins within $1 \mathrm{~m}$. If a group of dolphins was present, intensity of the whistle was only used to determine the whistling individual when one dolphin was close to the hydrophone $(<1 \mathrm{~m})$ and the rest of the group was further away ( $\geq 5 \mathrm{~m}$ ). Some previous studies have relied on only bubble streams to identify the vocalizing individuals $[12,13,14]$. However, our unique underwater viewing allowed us to note not only bubble streams, but also the directional orientation and proximity of individuals to the recording equipment and the lack of other dolphins at the safari park, Cisarua Bogor in Indonesia. Whistles were digitized from audio recordings using Raven Pro 1.5 software (Cornell University, Ithaca, NY, USA) at $44.1 \mathrm{kHz}$ sampling rate (Whistle 1, 2, 3, and 4). Data Analysis with Test F, All statistical tests were run with SPSS 14.0 software, and Noisy Time Domain were run with Matlab R 2008b

\section{Results and discussion}

Power Spectral Density (PSD) function to equalize the number of rows and columns of data matrix m-file of the results of the voice recording process. Power spectral density is a useful concept to determine the optimum frequency band of the signal transmission system. PSD is a variation of power (energy) as a function of frequency spectrum in the form of density estimated using FFT, PSD method is one of the modern spectral estimation technique proposed during this decade ([15] Figure 2 obtained that there are four whistle, the value of Power Spectral Density (PSD), which is found in the highest with $28.03 \mathrm{~dB} / \mathrm{Hz}$ are 
shown in green color on a whistle sound three at a time before eating pools show (indicated by black circles). The highest intensity values at an interval of $14642 \mathrm{~Hz}$ frequency-16000, while the lowest value that is currently on a whistle 4 with a frequency of $9300 \mathrm{~Hz}$ with a value of Power Spectral Density (PSD) $21.97 \mathrm{~dB} / \mathrm{Hz}$ are indicated by black lines. By looking at the pattern of spectral, whistle
3 has a sound frequency in the range of $14642-16000 \mathrm{~Hz}$ which is the highest compared to the intensity of the sound 1,2 , and 4 . The peak whistle sound are at 1 and 4 , the sound of the whistle 1 and 4 has a frequency range of peaks sound $14100-17000 \mathrm{~Hz}$ in frequency. Waveform whistle sound of dolphin in figure 1, and Power Sepctral Density ( PSD ) showed in figure 2 .
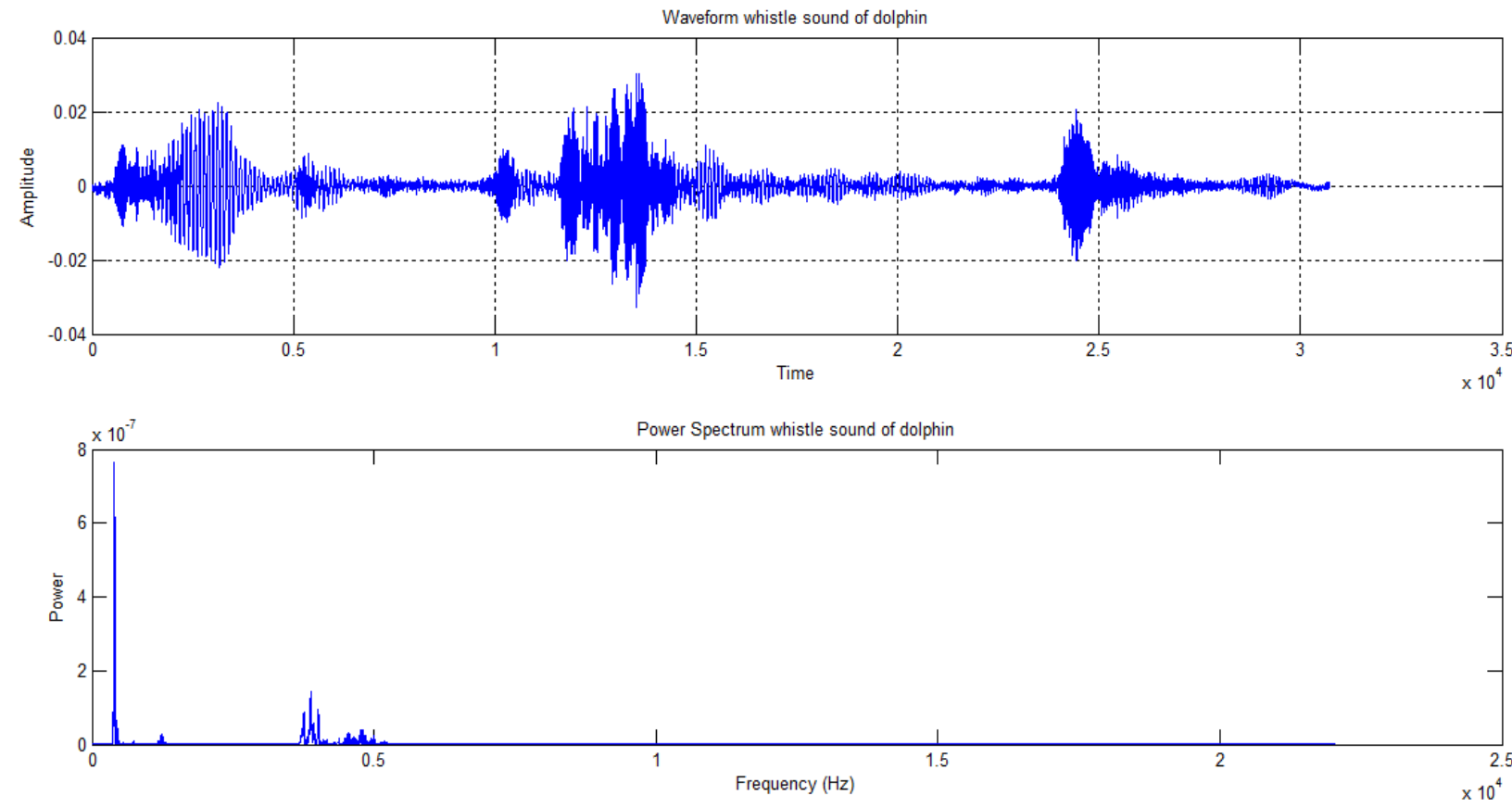

Figure 1. Waveform and Power Spectrum of dolphin

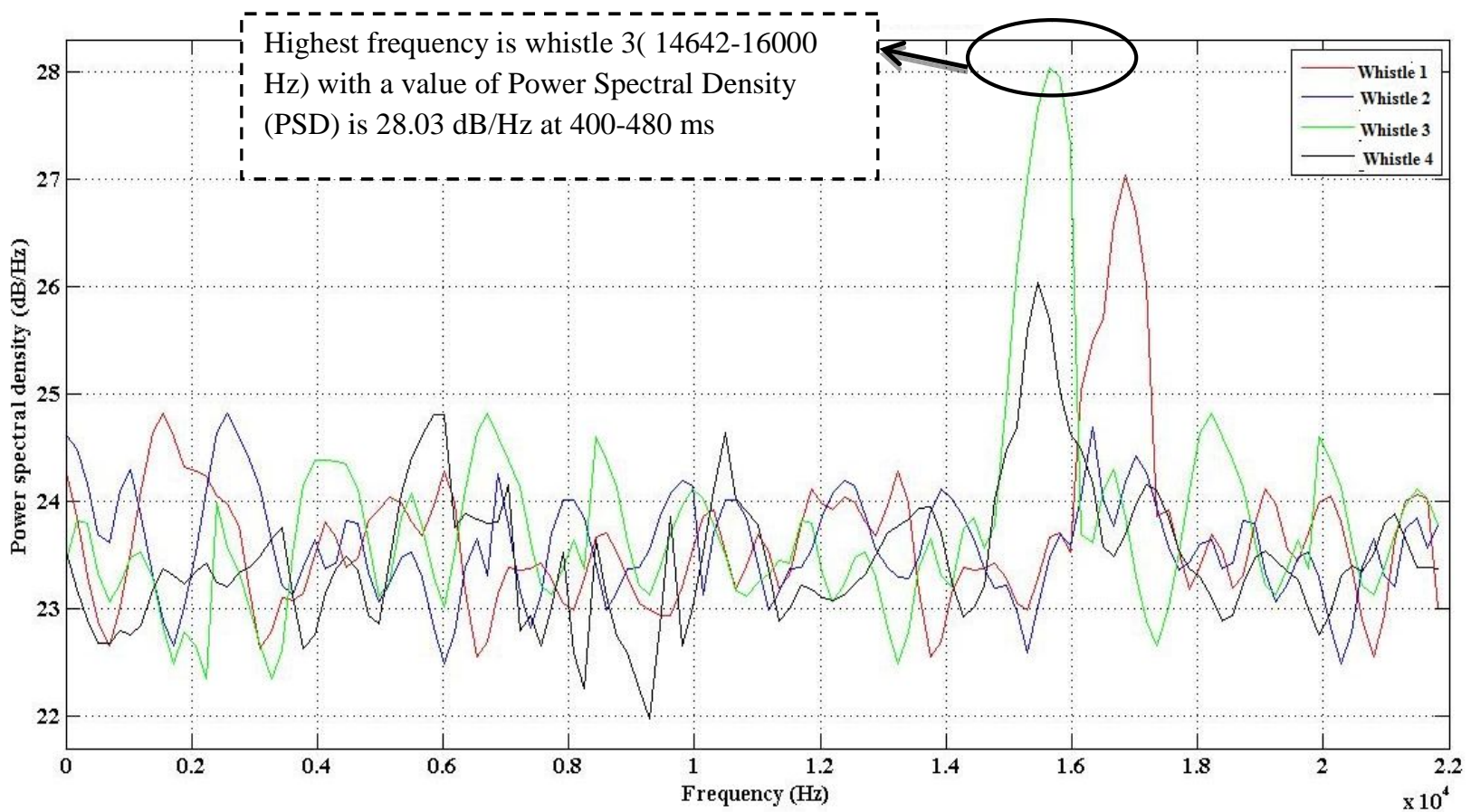

Figure 2. Power Spectral Density (PSD) whistle 1 before eating pool show 


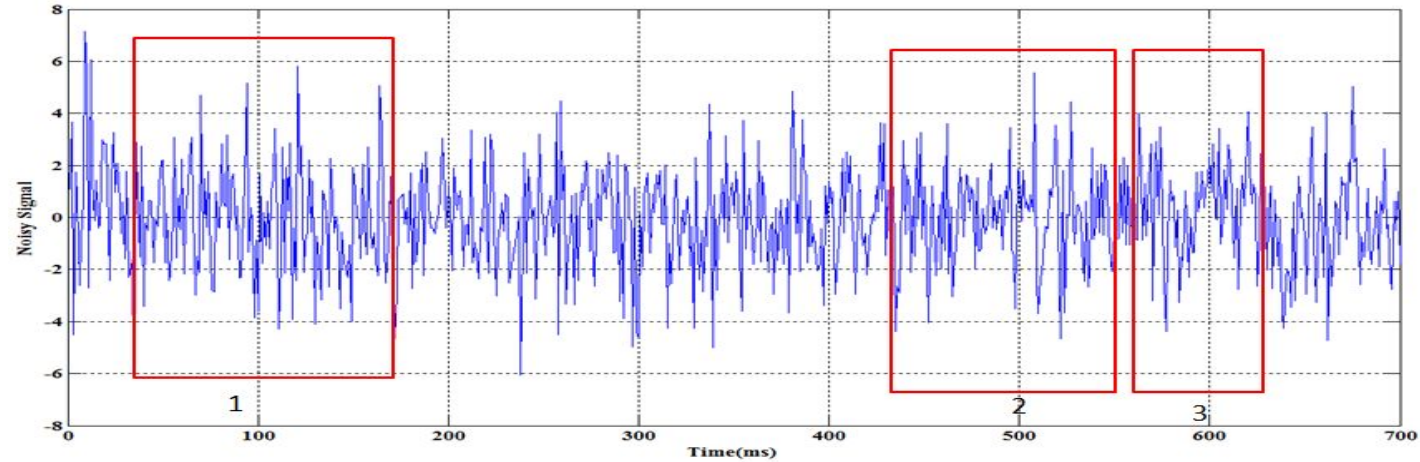

Figure 3. Noisy time domain whistle 1 before eating pool show

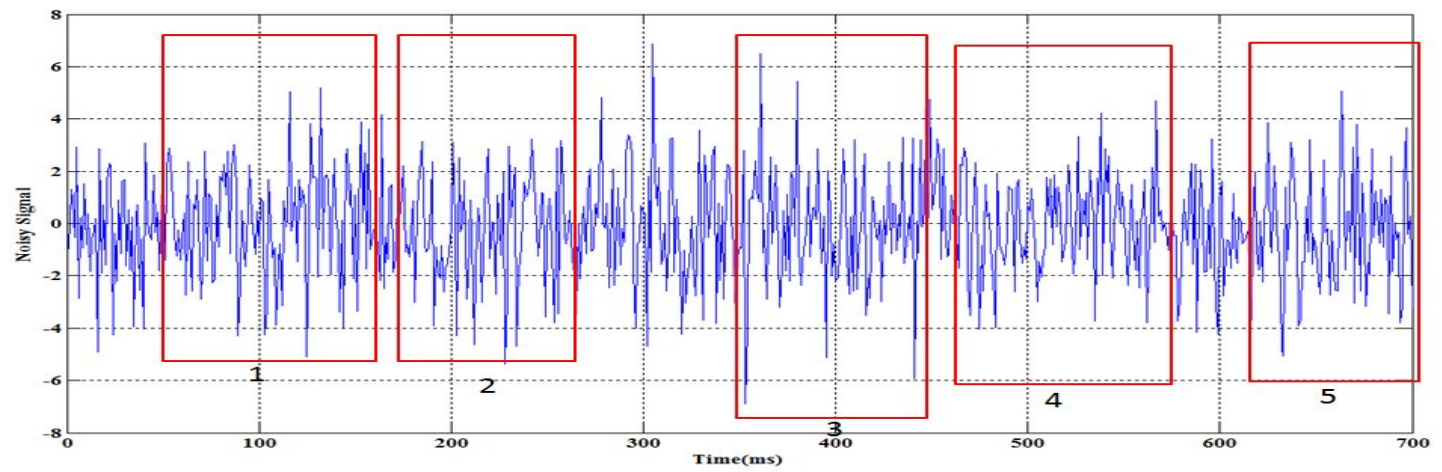

Figure 4. Noisy time domain whistle 2 before eating pool show

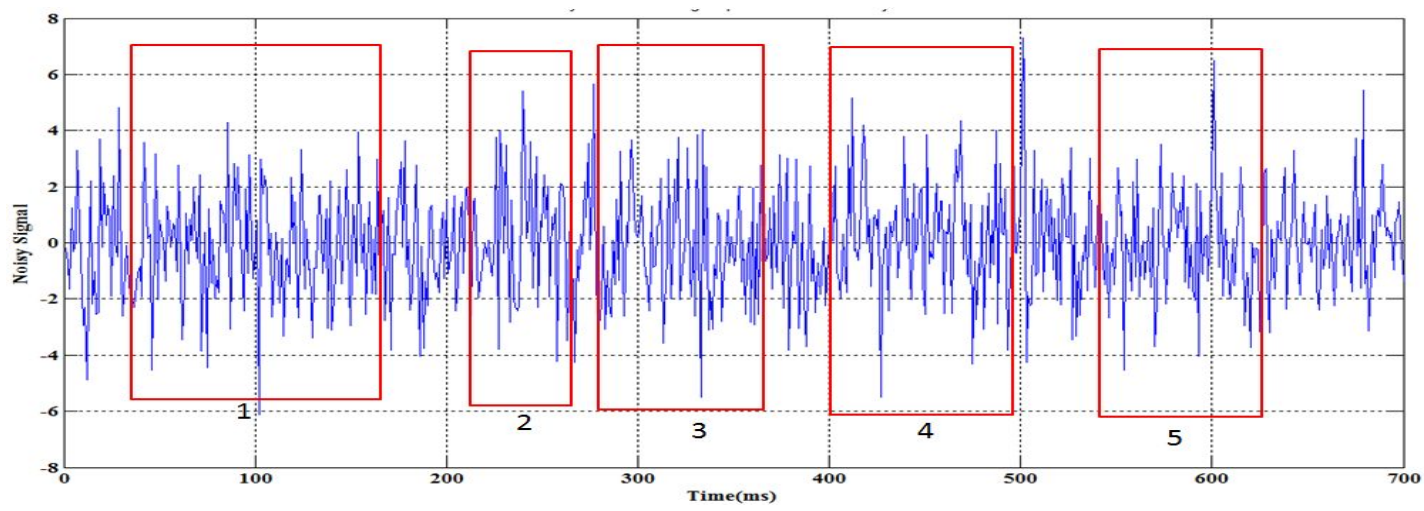

Figure 5 Noisy time domain whistle 3 before eating pool show

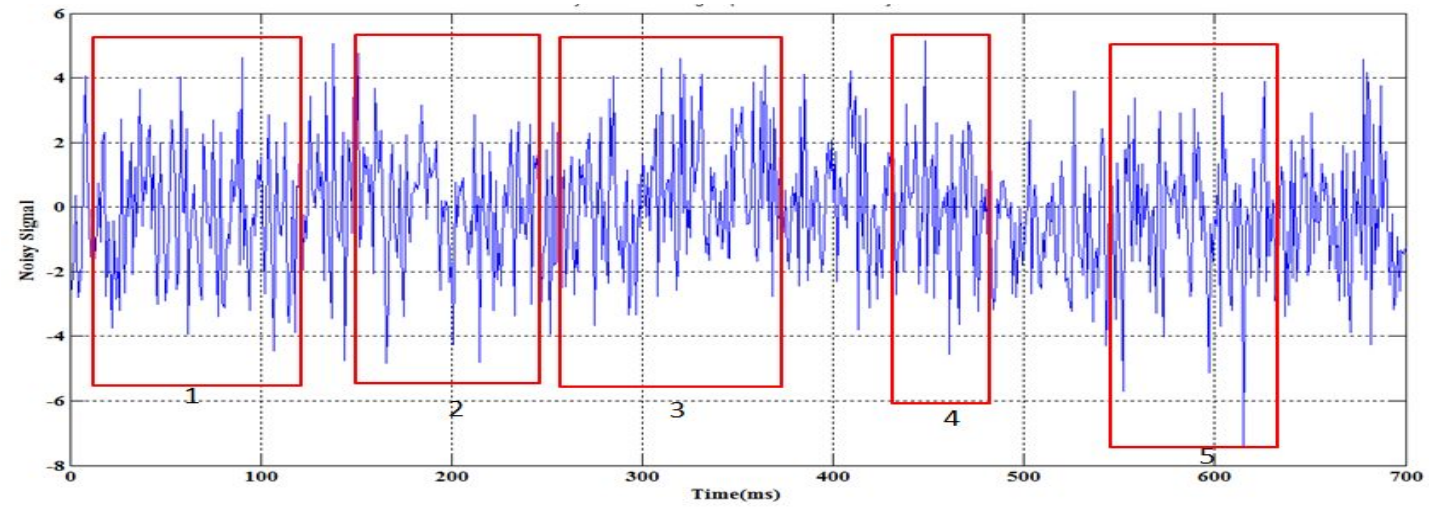

Figure 6. Noisy time domain whistle 4 before eating pool show 
Figure 3,4,5 and 6 have the highest peak value which is located at 2 and whistles whistle sound 3 with a noisy value of 6.2 at 370 and $600 \mathrm{~ms}$ contained in the pattern ke- 3 and the pattern of all five whistle sound (Figure 4 and 5). Results Noisy time domain has the same relationship with the original spectrum produced (Figure 4 and 5). Noisy lowest value in this is equal to -7.8 is at whistle sound 4 with time $620 \mathrm{~ms}$ on the 5 th pattern (Figure 7).

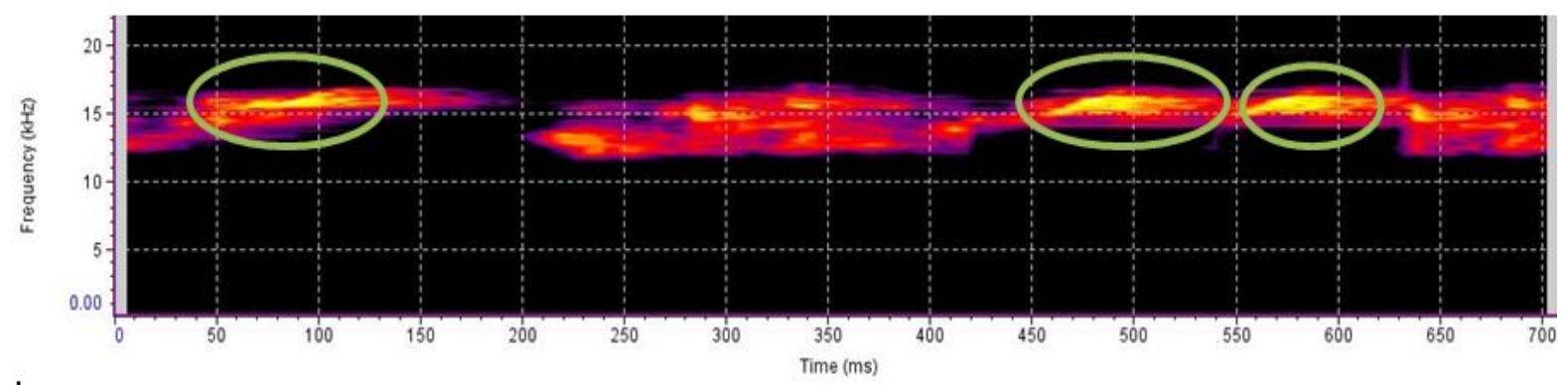

Figure 7. Spectogram whistle 1 before eating pool show

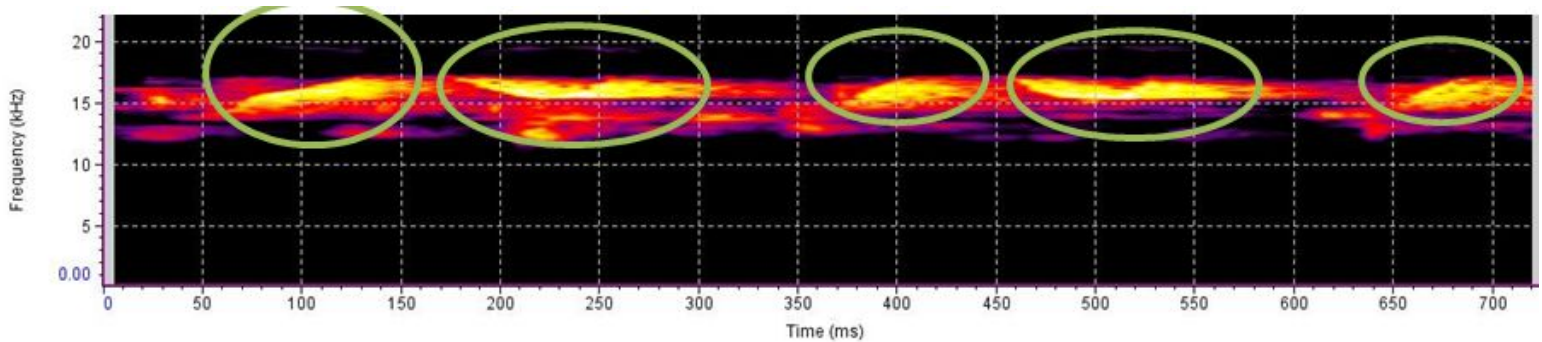

Figure 8. Spectogram whistle 2 before eating pool show

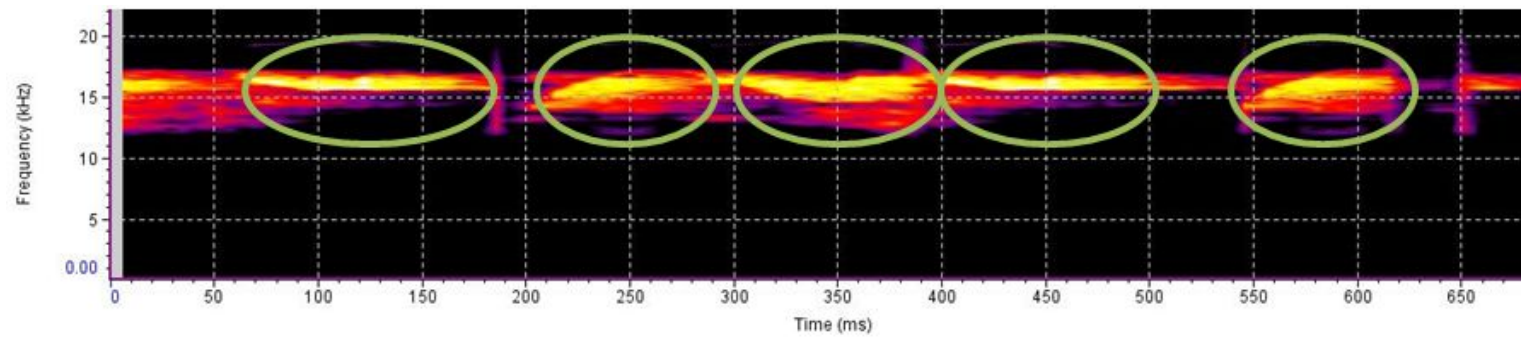

Figure 9. Spectogram whistle 3 before eating pool show

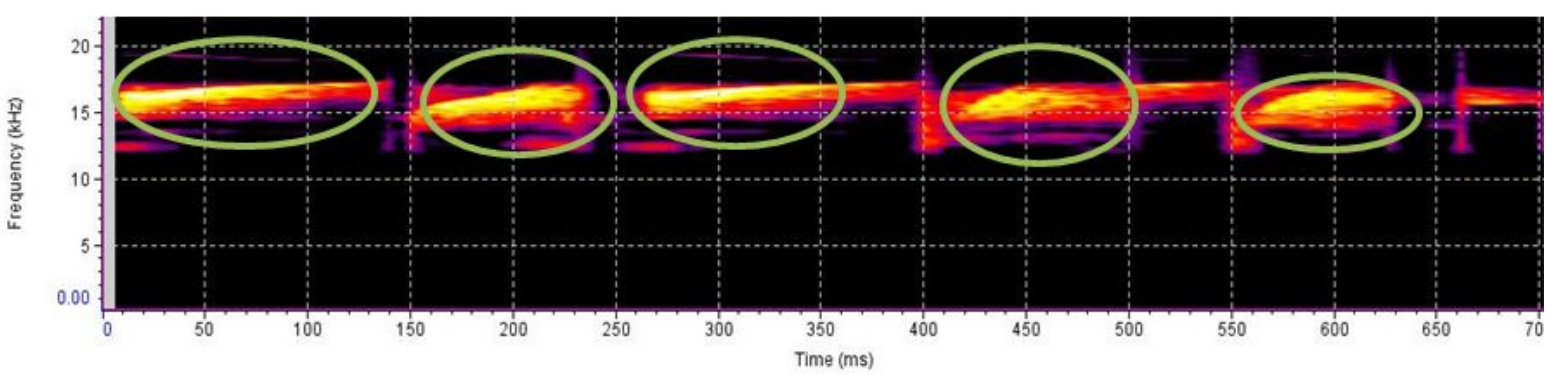

Figure 10. Spectogram whistle 4 before eating pool show

Figure 7 has 5 sound patterns that were at the time of 70-140 ms, ms 1190-260, 3900-440 ms, 460-560 $\mathrm{ms}$, and 6600-720 ms. In Figure 8 have a sound pattern 5 which is located at 70-160 ms, 230-260 ms, 280-300 $\mathrm{ms}, 400-480 \mathrm{~ms}$ and 560-620 ms. In Figure 9 also has five sound pattern with a time that is $20-100 \mathrm{~ms}, 150$ -
$270 \mathrm{~ms}, 270-350 \mathrm{~ms}, 440-470 \mathrm{~ms}$ and $560-630 \mathrm{~ms}$. Shape pattern is marked by a green circle, with a maximum time whistle sound original 1,2, 3, 4 which is $700 \mathrm{~ms}$. Average frequency in each pattern shown on the Figure is located at $15000-16000 \mathrm{~Hz}$ frequency range. From the results obtained can be identified as 
the whistle sounds emanating from dolphins by calculating or viewing range interval whistle sound itself [16]. Highest intensity on a whistle 4 can also be affected by the position with the position of the dolphin is horizontal, because according to [17] movement in the vertical position will probably affect the magnitude of the sound and the inconsistency of the sound emitted by the dolphins using conservation methods energy obtained by dolphins, and will require a lot of energy to expend greater voice intensity value.

Table 1. F Test before eating pool show

\begin{tabular}{cccccc}
\hline \multirow{2}{*}{ No } & Time (ms) & \multicolumn{4}{c}{ Whistle Sound Before Eating Pool Show } \\
\cline { 3 - 6 } & & Whistle1 & Whistle2 & Whistle3 & Whistle4 \\
\hline 1 & 100 With 200 & $*$ & - & - & - \\
2 & 200 With 300 & - & - & $*$ & - \\
3 & 300 With 400 & $*$ & $*$ & - & - \\
4 & 400 With 500 & - & - & $*$ & $*$ \\
5 & 500 With 600 & - & - & $*$ & $*$ \\
6 & 600 With 700 & $*$ & $*$ & - & $*$ \\
\hline
\end{tabular}

-)No significant effect (Thank H0) (Fhit <Ftabel)

*)Significant (Reject H0) (Fhit> Ftabel)

F test based on Table 1 whistle sound 1 at 100 to 200, 200 to $300 \mathrm{~ms}, 300 \mathrm{~ms}$ to 400,400 to 500,500 to $600 \mathrm{~ms}$, and 600 to $700 \mathrm{~ms}$ has a $\mathrm{P}$ value $>0.001$. 2 whistle sound at a time of 100 to 200,200 to $300 \mathrm{~ms}$, $300 \mathrm{~ms}$ to 400,400 to 500,500 to $600 \mathrm{~ms}$, and 600 to $700 \mathrm{~ms}$ has a $\mathrm{P}$ value $>0.001$. Whistle 3 at 100 to 200 , 200 to $300 \mathrm{~ms}, 300 \mathrm{~ms}$ to 400,400 to 500,500 to 600 $\mathrm{ms}$, and 600 to $700 \mathrm{~ms}$ has a $\mathrm{P}$ value $>0.001$. 4 whistle sound at 200 to $300 \mathrm{~ms}, 400$ to 500 , and 500 to 600 ms.memiliki $\mathrm{P}>0.001$, while 100 to 200,300 to 400 , 600 to $700 \mathrm{~ms}$ has a value of $\mathrm{P}<0.001$. F-test nothing in common (Heterogeneous) whistle 1, 2, 3, and 4, while the value of $P>0.001$ contained on whistle sound 1,2 , and 3 , while the sound of the whistle $4 \mathrm{p}<0.001$ at 300 with 400 to $700 \mathrm{~ms}$ and $600 \mathrm{~ms}$. Behaviour of male dolphin bottle nose showed by figure 11 .
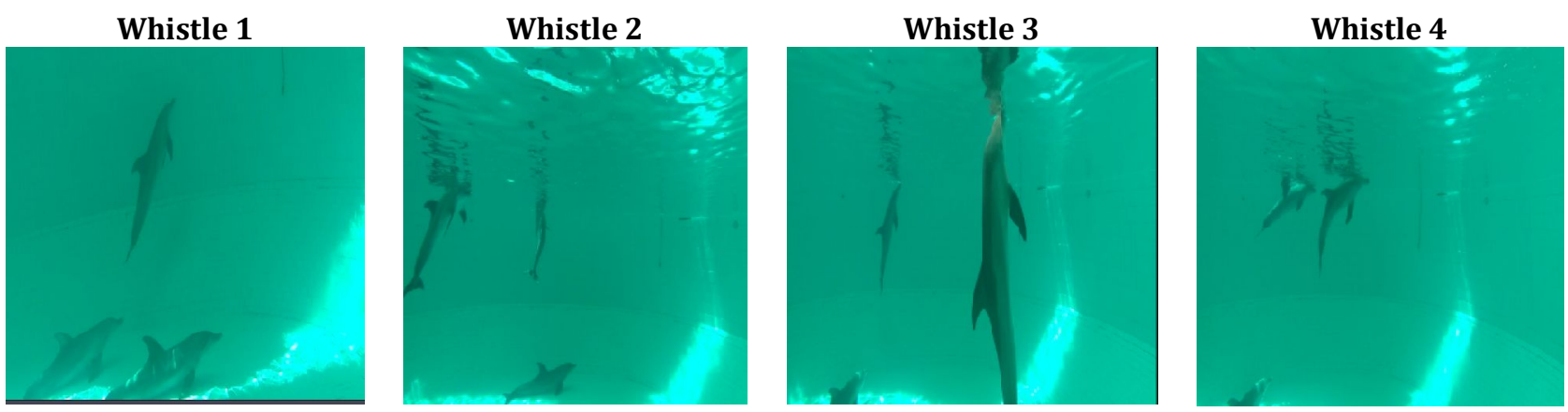

Figure 11. Behaviour of male dolphin bottle nose before eating pool show

The position of the dolphins in the pool show before dining on whistle 1, 2 dolphins were bottom of the pool and 1 tail on the surface of a pond, a whistle 22 dolphins on the surface, whistle 3 and 4 have the same position with a whistle 2. dolphins are more dominant on the surface at the time before eating, and behavior of dolphins is more inclined to the surface of the pool. The position of the dolphins is very influential on the resulting frequency $[18,5,6]$.

\section{Conclusions}

Male dolphin bottle nose (Tursiop aduncus) are maintained in an physiotherapy and show, Safari Park Indonesia Cisarua, Bogor has a Power Spectral Density (PSD), value Noisy Time Domain have differences with each other and have a lot of different sound patterns. In general, there are real differences are evidenced by $\mathrm{F}$ test on a range of time at each whistle on physiotherapy and swimming pool show in the time before meals. The position of dolphins in a pool show more dominant and often in the bottom of the pool, the position of the dolphins affect the frequency.

\section{Acknowledgments}

I express my sincere thanks to the referees for their valuable suggestions to improve the paper in present form. The authors are grateful to safari park which already allow authors to collect data during the research. The authors also thanks to lecturers Dr. Ir Sri Pujiyati, Dr. Ir. Totok Hestirianoto and my research associates are Pratiwi Dwi Wulandari BSc in Department Marine Science and Technology, Bogor Agricultural University . 


\section{References}

[1] Bebus Sara. E \& Herzing L. Denise Mother-Offspring Signature Whistle Similarity and Patterns of Association in Atlantic Spotted Dolphins (Stenella frontalis) Scinow Publications Ltd. ABC 2015 2(1):71-87 Animal Behavior and Cognition

[2] Azzolin, M. Papale, E., Lammers, M. O. Gannier, A., \& Giacoma, C. 2013 Geographic variation of whistles of the striped dolphin (Stenella coeruleoalba) within the Mediterranean Sea. The Journal of the Acoustical Society of America, 134, 694

[3] Papale, E. Azzolin, M., Cascão, I., Gannier, A., Lammers, M. O., Martin V. M. \& Giacoma, C. 2013. Macro-and micro-geographic variation of short-beaked common dolphin's whistles in the Mediterranean Sea and Atlantic Ocean. Ethology Ecology \& Evolution, (ahead-of-print), $1-13$

[4] Janik, V. M, and Slater, P. J. 1998. Context-specific use suggests that bottlenose dolphin signature whistles are cohesion calls, Animal Behav. 56, 829-838.

[5] Lubis, M.Z, Pujiyati.Sri, Hestirianoto.Totok. 2016a. Bioacoustic Characteristic of Male Dolphins Bottle Nose (Tursiops aduncus ) International Journal of Scientific Engineering and Technology ISSN:2277-1581,Volume No.5 Issue No.1, pp: 44-49

[6] Lubis, M.Z, Pujiyati.Sri, Hestirianoto.Totok, Wulandari P.D. 2016b. Bioacoustic Characteristics of Whistle Sounds and behaviour of male Indo-Pacific bottlenose dolphins (Tursiops aduncus) in Indonesia. International Journal of Scientific and Research Publications, ISSN 2250-3153, Volume 6, Issue 2.

[7] Janik, V. M. 2000. Whistle matching in wild bottlenose dolphins (Tursiops truncatus). Science 289:1355-1357.

[8] Simmonds J. \& MacLennan D. 2005. Fisheries Acoustics: Theory and Practice, second edition. Blackwell.
[9] Popper, A. N. And Hasting M.C. 2009. The effects of anthropogenic sources of sound on fishes. Journal of Fish Biology 75, 455-489

[10] Caldwell, M. C. \& Caldwell, D. K. 1965 Individualized whistle contours in bottlenosed dolphins (Tursiops truncatus). Nature 207, 434-435.

[11] Krauss,T.P.,L. Shure and J.N.Little 1995. Signal Processing Toolbox: For Use with Matlab. The Mathworks, Inc

[12] McCowan, B., \& Reiss, D. (1995a). Quantitative comparison of whistle repertoires from captive adult bottlenose dolphins (Delphinidae, Tursiops truncatus): A re-evaluation of the signature whistle hypothesis. Ethology, 100, 194-209. doi: 10.1111/j.14390310.1995.tb00325.x

[13] McCowan, B., \& Reiss, D. (1995b). Whistle contour development in captive-born infant bottlenose dolphins (Tursiops truncatus): Role of learning. Journal of Comparative Psychology, 109, 242-260. doi: 10.1037/0735-7036.109.3.242

[14] Reiss, D. (1988). Observations on the development of echolocation in young bottlenose dolphins. In P. E. Nachtigall \& P. W. B. Moore (Eds.) Animal sonar (pp. 121-127). New York: Plenum Publishing.

[15] Stoica. P and R.L. Moses. 1997. Introduction to spectral Analysis. New Jersey: Prentice Hall Inc

[16] Cahill, T.2000. Dolphins National Geografic Society. Washington DC.

[17] Dudzinski, K. M., Sakai, M., Masaki, K., Kogi, K., Hishii, T., \& Kurimoto, M. 2003. Behavioural observations of bottlenose dolphins towards two dead conspecifics. Aquatic Mammals, 29, 108- 116.

[18] Gridley, T., Cockcroft, V. G., Hawkins, E. R., Blewitt, M. L., Morisaka, T., \& Janik, V. M. (2014). Signature whistles in free-ranging populations of Indo-Pacific bottlenose dolphins, Tursiops aduncus. Marine Mammal Science, 30, 512-527. doi: 10.1111/mms.12054.

[19] Brook,D. and R.J. Wynne. 1991. Signal Processing: Principples and Applications. Edward Arnold, a division of Hodder and Stoughton Limited, Mill Road, Dunton Green. Great Britain. 